\title{
Sedation and weaning from mechanical ventilation: time for 'best practice' to catch up with new realities?
}

\author{
Giorgio Conti ${ }^{1 *}$, Jean Mantz ${ }^{2}$, Dan Longrois ${ }^{3}$ and Peter Tonner ${ }^{4}$
}

\begin{abstract}
Delivery of sedation in anticipation of weaning of adult patients from prolonged mechanical ventilation is an arena of critical care medicine where opinion-based practice is currently hard to avoid because robust evidence is lacking. We offer some views on this subject, hoping to stimulate debate among colleagues.
\end{abstract}

Keywords: Intensive care, Mechanical ventilation, Sedation, Weaning

\section{Introduction}

Although even brief scrutiny of MEDLINE and ClinicalTrials.gov reveals that vigour and ingenuity are being directed towards evaluating every aspect of intensive care unit (ICU) medicine, it remains the case that a comprehensive base of evidence is not available for every aspect of practice. In the absence of conclusive evidence, well-founded opinions retain a role in providing a framework for clinical reasoning and decision making.

Whether and how to deliver sedation in anticipation of weaning of adult patients from prolonged mechanical ventilation is one arena where such opinion-based medicine is called for as new methods and resources emerge, as the limitations of some established practices are better appreciated and as we wait for a new generation of randomized controlled trials (RCTs) to provide definitive evidence. In this paper we offer some views on this subject, hoping to stimulate debate among colleagues.

Issues for consideration in this context include:

(1) Definition of weaning and the distribution of weaning experience

(2) Aspects of current practice that should be discarded

(3) Criteria for weaning

(4) Influence of the type/quality of sedation on the success/failure of weaning in ICU patients

\footnotetext{
* Correspondence: g.conti@rm.unicatt.it

'Department of Intensive Care and Anesthesiology, Università Cattolica del Sacro Cuore, Rome, Italy

Full list of author information is available at the end of the article
}

(5) Weaning in the context of cooperative sedation (a concept unknown when several pivotal RCTs on weaning were performed).

\section{Defining weaning}

Weaning is the liberation of a patient from mechanical ventilatory support. As such it is a process not an outcome and a process that starts when the decision is taken to intubate a patient. Weaning is thus not the same thing as extubation; rather, extubation may be seen as the culmination of the weaning process.

Maintaining patients on the weaning pathway requires a holistic approach that prioritizes the best preservation of respiratory muscles without excessive stress to patients, maintenance of haemodynamic stability, nutrition and electrolyte status, and attention to patient's motivation and engagement.

A corollary of this definition of weaning is that noninvasive ventilation (NIV) can be an important and valuable part of the weaning process and an adjunct to extubation. This is demonstrably the case in chronic obstructive pulmonary disease (COPD), as shown in several RCTs [1-3]. NIV is often applied in non-COPD cases of hypoxaemic respiratory failure, though as yet without the substantiation of major RCTs $[4,5]$.

\section{The distribution of weaning experience}

The general pattern of weaning described by Boles et al. [6] remains a broadly accurate summary of routine experience. Thus some $70 \%$ of mechanically ventilated patients 
are successfully weaned at the first attempt. Of the remaining 30\%, about $25 \%$ experience 'difficult' weaning (defined as failure the first time but success within 1 week of the first attempt) and 5\% experience prolonged weaning (defined as $>1$ week with repeated attempts).

The confirmation by the Spanish Lung Failure Collaborative Group that ventilator support can be successfully discontinued in two-thirds of ventilated patients after a 2-h spontaneous breathing trial (SBT) is also compatible with this distribution [7].

\section{Aspects of current practice that should be discarded}

Three elements of what might be termed the classic weaning schedule are either unnecessary or redundant and should be discarded.

\section{Predictors of weaning}

None of the claimed predictors of weaning, whether used alone or in combination, are reliably predictive of weaning success and no time or effort should be wasted on monitoring them for that purpose. This lack of reliability is evident for unitary criteria such as vital capacity, maximal inspiratory pressure and minute ventilation [8-10] and for the respiratory frequency to tidal volume (f/VT) ratio [11], which at one time seemed the most promising practical predictive instrument.

\section{The spontaneous awakening trial}

The adoption by ICUs (certainly in Europe) of awake, cooperative sedation as the standard of care means that the spontaneous awakening trial [12] is now largely an anachronism, relevant only to those narrow sectors of the ICU population where deep sedation is still needed (e.g. traumatic brain injury [TBI] or acute respiratory distress syndrome [ARDS] patients or those receiving neuromuscular blockers [NMBs]). Outside those areas, few ICU patients are too ill to be awake with sedation provided proper attention is given to their needs for analgesia.

\section{Early tracheotomy}

Given that some $70 \%$ of patients can expect to be weaned successfully at the first attempt, there is no argument for a generalized use of early tracheotomy as a prelude or adjunct to weaning $[13,14]$. There may be exceptions to that verdict for brain-injured patients or multiple trauma victims.

\section{Criteria for weaning}

The central question in weaning is when to complete the return to unsupported spontaneous breathing. Readiness should be monitored by 'dynamic assessment', anchored by a series of key considerations that emphasize trend and stability, rather than by rigid thresholds.
1. Resolution of the underlying cause of acute respiratory failure

2. Haemodynamic stability, defined as no need for vasoactive/inotropic drugs

3. Adequate neurological status, defined as: Glasgow Coma Scale score $>8^{\mathrm{a}}$ or, if sedated, target Richmond Agitation-Sedation Scale score in the range -2 to 0 achieved with minimal sedation

4. Preferably absence of fever (defined as temperature $\left.<38^{\circ} \mathrm{C}\right)^{\mathrm{b}}$

5. Adequate gas exchange, as indicated by a partial pressure of oxygen:fraction of inspired oxygen ratio $>200$ with a positive end-expiratory pressure of $5 \mathrm{cmH}_{2} \mathrm{O}$

6. Partial pressure of carbon dioxide adjusted to bring blood $\mathrm{pH}$ into the normal range [7].

\section{Weaning in the context of cooperative sedation}

Current guidelines on weaning rest very considerably on the findings of RCTs performed during the last decade of the $20^{\text {th }}$ century. One of those studies demonstrated that immediate extubation after a successful SBT accelerates weaning and reduces the duration of mechanical ventilation [7]. Two other studies showed that the ability to breathe spontaneously can be adequately tested by a trial with either T-tube or pressure support of $7 \mathrm{cmH}_{2} \mathrm{O}$ lasting either 30 or $120 \mathrm{~min}$ [15]. Two randomized studies found that synchronized intermittent mandatory ventilation is the worst method of weaning in difficult-to-wean patients $[7,16]$.

However, all those studies required patients to be sedative-free before weaning was attempted. That was a reasonable stipulation given the nature of the sedatives and the sedation protocols then prevailing. In view of the current widespread emphasis on cooperative sedation - a concept undeveloped at the time of those trials - and the increased availability of sedatives free from adverse effects on respiration, there is an evident need for new studies analysing the effects of these new sedation paradigms on the weaning process.

Until such studies are completed, the evidence base for the optimized management of weaning is incomplete. However, it is possible to propose a framework of guidance for current practice.

- Most patients who require sedation in the context of weaning are likely to be among the $30 \%$ or so of patients not weaned successfully at the first attempt. Ongoing sedation in the context of weaning will thus be confined most likely to a minority of patients.

- 'Sedation' should be described and configured as 'analgo-sedation'. This word highlights the primacy of pain relief for the delivery of patient comfort before, during and after weaning. 
- Minimum sedation is the prerequisite for weaning and should be regarded in any case as the default target in the absence of special circumstances such as severe ARDS, TBI or use of NMBs.

- Use of longer-acting sedatives should be avoided. There should be, in particular, a strong presumption against the use of benzodiazepines, which ought now to be regarded as drugs of last resort in most situations. This latter conclusion is aligned with and draws support from the recent retrospective analyses of Fraser et al. [17].

- Rapid or abrupt termination of an established sedative regimen should be avoided, especially when longer-acting sedatives have been used.

- Step-down sedation, for example substituting propofol for a benzodiazepine, may be needed for patients who have been maintained on longer-acting agents.

- Monitoring of minimally sedated awake, cooperative patients for pain, agitation and delirium (both hyperand hypo-active) should be systematically conducted at least once a day as part of the weaning routine. (This need not take very long.) When detected, the first response must be to correct the primary cause, not to obscure it with sedation. The general principles and practical guidance enumerated by Barr and colleagues [18] provide the basis for current best practice in this area, but it should be noted that many of those recommendations are based on limited evidence, especially in the area of ICU delirium.

- Identification and effective treatment of pain is a priority, including pain as the cause of agitation. Delirium (in whatever form) is often associated with pain or agitation and in these cases it will likely respond to measures to relieve pain and agitation. We concur with Barr et al. [18] in regarding the Confusion Assessment Method for The Intensive Care Unit and the Intensive Care Delirium Screening Checklist as currently the best instruments for identification of delirium, combining practicability with reliability and accuracy (see also Neto et al. [19]). (It is again important to note that at the time when some major RCTs on weaning were performed, even the concept of 'delirium' was poorly described and not incorporated in the decision analysis.)

- The long-term impact of delirium on the trajectory of an ICU patient remains a matter of debate, but a growing body of evidence supports the assertion that delirium is associated with impaired post-ICU cognitive function [20]. There is more certainty about its adverse impact on the patient's course in the ICU and in particular on the duration of mechanical ventilation. Attention to delirium is thus an essential feature of preparations for weaning, including all aspects of sedation practice. Barr et al. [18] identify continuous infusion of dexmedetomidine as the only pharmacological measure likely to reduce the duration of delirium.

- Because many patients who need sedation as an adjunct to weaning will have experienced prolonged or difficult weaning, effects of sedatives on respiratory function are an important consideration.

- Sedatives that act via $\gamma$-aminobutyric acid (GABA)-ergic pathways (e.g., benzodiazepines, opioids and propofol) adversely affect respiratory drive and/or timing. This aspect is very important, as sedation with GABA-ergic and/or morphine-like agents can represent per se a source of patient-ventilator asynchrony, prolonging the need for ventilatory support [21].

- Alpha 2 -agonists do not adversely affect respiratory drive and/or timing.

A range of sedative regimens are in use in Europe, reflecting differing circumstances, priorities or perspectives at national or sub-national levels. It is, therefore, infeasible to recommend a single agent or regimen, but for patients undergoing difficult or prolonged weaning there should be a presumption in favour of short-acting sedatives delivered as a continuous infusion. Remifentanil and dexmedetomidine are particularly well suited to that requirement and can be relied on to deliver comfort to patients emerging into spontaneous breathing while also meeting the goal of minimal/awake sedation. Low-dose morphine in the manner advocated by Strøm et al. [22] may also be considered, though there is a widespread assumption (not yet formally tested) that this approach requires a very low (probably 1:1) staff:patient ratio to enable it to be implemented consistently successfully.

Remifentanil should be used as a very low-dose continuous infusion (e.g. $0.05 \mu \mathrm{g} / \mathrm{kg} / \mathrm{min}$ ) to avoid possible adverse effects on respiration [23].

Dexmedetomidine appears to be particularly well suited to the needs of patients receiving NIV as part of a step-down ventilation strategy or for the prevention (but not treatment) of post-extubation weaning failure. One rationale for this application is that NIV enables some patients to be extubated sooner than might be possible if they were being restored directly from mechanically supported to fully unsupported breathing. Patients proceeding to NIV may, therefore, be extubated sooner and/or at a deeper level of sedation than if they were being restored to unsupported breathing. Dexmedetomidine-based sedation during NIV may, therefore, be used as a step-down measure replacing longer-acting sedatives and to prepare the patient for the final phase of weaning by establishing conscious sedation, just as NIV itself is used as a step-down ventilation measure prior to extubation. 
Epstein [24] and Burns and colleagues [25] have noted that the evidence from randomized trials of the benefit of NIV as an aid to weaning or to prevent post-extubation failure is substantially limited to acute-on-chronic respiratory failure, most obviously COPD. Whether or not using NIV under present conditions reflects this limitation and whether an emphasis on newer sedatives such as dexmedetomidine might enhance the success of NIV in this context or expand the range of valid clinical situations in which NIV is advantageous are matters deserving of early investigation.

Other situations in which dexmedetomidine is strongly to be favoured include:

- Patients at risk for weaning syndrome and/or delirium, or otherwise vulnerable to mental deterioration for non-organic reasons

- Patients experiencing sleep deprivation. (Dosages may be adjusted throughout the 24-h cycle to help restore a more normal sleep-wake pattern, in the wider context of measures to promote/protect sleep. Though inter alia see Tamrat et al. [26] for a recent systematic review of non-pharmacological measures to promote sleep. The broad conclusion of that work is that there is a lack of good-quality evidence to guide practice in this arena.)

\section{Conclusions}

The time has probably come to revisit all aspects of weaning and weaning protocols in view of recent transformation in sedative practice for critically ill patients [18]. Considering the interrelation between sedation and weaning it is increasingly difficult to justify persisting with practice based on experience and conclusions derived from sedation studies performed with drugs that possess important pharmacological drawbacks and which increasingly are regarded as seldom appropriate except as agents of last resort in an era when little if any use was made of sedation scales and when the significance (or even existence) of delirium was greatly under-appreciated and formed no part of clinical reasoning or procedure.

It is time that the evidence base for sedation and weaning was enlarged to reflect the new realities already in force in many countries. In the short term there should be a concerted effort to address the many areas of practice where the authors of the pain, agitation, and delirium (PAD) guidelines [18] could find either no evidence better than grade $\mathrm{C}$ or no evidence at all. As a longer-term strategy we propose that research should aim to provide evidential support for all recommendations of the 2013 PAD guidelines to at least level 1B.

\section{Endnotes}

${ }^{a}$ Weaning success rates in TBI and neurocritical patients are not always nil. This implies that the minimum level of consciousness required for successful weaning may be lower than is generally acknowledged; however, these data do not justify accepting worse than the best achievable level of awareness in non-TBI patients. ${ }^{\mathrm{b}}$ Temperature $>38^{\circ} \mathrm{C}$ is not an absolute barrier if the fever is of non-infectious origin. In such cases, the delay implied in waiting several days for temperature to fall below $38^{\circ} \mathrm{C}$ may itself be an outcome worth trying to avoid.

\section{Competing interests}

GC reports honoraria for lectures from Orion Pharma (Italy), Covidien and Sylcomed. The Catholic University of Rome has received an institutional research grant from Orion Pharma.

JM reports honoraria from Orion Pharma as a guest lecturer at international symposia in intensive care.

DL reports honoraria from Orion Pharma for guest lectures at international symposia in anaesthesia and intensive care, and editorship of intensetimes, an electronic journal supported by Orion Pharma.

PT reports honoraria for lectures at national and international symposia from Orion Pharma, Abbvie, Baxter, Bard, B. Braun and Ratiopharm.

\section{Authors' contributions}

All authors contributed directly to the discussions that gave rise to this article. All authors read and approved the final manuscript.

\section{Acknowledgements}

The authors thank Riku Aantaa (Turku, Finland), Alexander Scott (Leeds, UK), Dorte Bulow Keld (Aarhus, Denmark), Alberto Sandiumenge (Tarragona, Spain), Ilona Bubek (Budapest, Hungary) and Per-Olof Joachimson (Uppsala, Sweden) for their contributions to the roundtable debate that provided the intellectual framework of this article. The roundtable was made possible through the financial support of Orion Pharma, Espoo, Finland.

Preparation of this manuscript was assisted by Hughes associates, Oxford, UK, on behalf of Orion Pharma.

\section{Author details}

'Department of Intensive Care and Anesthesiology, Università Cattolica del Sacro Cuore, Rome, Italy. ${ }^{2}$ Anesthesiology Department, Beaujon Hospital, AP-HP, Université Paris-Diderot, Paris, France. ${ }^{3}$ Département d'Anesthésie Réanimation Chirurgicale, Hôpital Bichat-Claude Bernard, Université

Paris-Diderot, Hôpitaux Universitaires Paris Nord Val de Seine, Paris, France ${ }^{4}$ Department of Anesthesiology and Intensive Care Medicine, Emergency Medicine Hospital Links der Weser GmbH, Bremen, Germany.

Received: 10 May 2014 Accepted: 30 July 2014

Published: 29 August 2014

\section{References}

1. Nava S, Ambrosino N, Clini E, Prato M, Orlando G, Vitacca M, Brigada P, Fracchia C, Rubini F: Noninvasive mechanical ventilation in the weaning of patients with respiratory failure due to chronic obstructive pulmonary disease. A randomized, controlled trial. Ann Intern Med 1998, 128:721-728.

2. Girault C, Daudenthun I, Chevron V, Tamion F, Leroy J, Bonmarchand G: Noninvasive ventilation as a systematic extubation and weaning technique in acute-on-chronic respiratory failure: a prospective, randomized controlled study. Am J Respir Crit Care Med 1999, 160:86-92.

3. Ferrer M, Esquinas A, Arancibia F, Bauer TT, Gonzalez G, Carrillo A, Rodriguez-Roisin R, Torres A: Noninvasive ventilation during persistent weaning failure: a randomized controlled trial. Am J Respir Crit Care Med 2003, 168:70-76.

4. Vaschetto R, Turucz E, Dellapiazza F, Guido S, Colombo D, Cammarota G, Della Corte F, Antonelli M, Navalesi P: Noninvasive ventilation after early extubation in patients recovering from hypoxemic acute respiratory failure: a single-centre feasibility study. Intensive Care Med 2012, 38:1599-1606.

5. Burns KEA, Meade MO, Premji AC, Adhikari NKJ: Noninvasive ventilation as a weaning strategy for mechanical ventilation in adults with respiratory failure: a Cochrane systematic review. CMAJ 2014, 186:E112-E122. 
6. Boles JM, Bion J, Connors A, Herridge M, Marsh B, Melot C, Pearl R, Silverman $\mathrm{H}$, Stanchina M, Vieillard-Baron A, Welte T: Weaning from mechanical ventilation. Eur Respir J 2007, 29:1033-1056.

7. Esteban A, Frutos F, Tobin MJ, Alía I, Solsona JF, Valverdú I, Fernández R, de la Cal MA, Benito S, Tomás R: A comparison of four methods of weaning patients from mechanical ventilation. Spanish Lung Failure Collaborative Group. N Engl J Med 1995, 332:345-350.

8. Gandía F, Blanco J: Evaluation of indexes predicting the outcome of ventilator weaning and value of adding supplemental inspiratory load. Intensive Care Med 1992, 18:327-333.

9. Sassoon $\mathrm{CSH}$, Mahutte CK: Airway occlusion pressure and breathing pattern as predictors of weaning outcome. Am Rev Respir Dis 1992, 148:860-866.

10. Yang $\mathrm{K}$, Tobin MJ: A prospective study of indexes predicting outcome of trials of weaning from mechanical ventilation. N Engl J Med 1991, 24:1445-1450.

11. Conti G, Montini L, Pennisi MA, Cavaliere F, Arcangeli A, Bocci MG, Proietti R Antonelli M: A prospective, blinded evaluation of indexes proposed to predict weaning from mechanical ventilation. Intensive Care Med 2004, 30:830-836.

12. Kress JP, Pohlman AS, O'Connor MF, Hall JB: Daily interruption of sedative infusions in critically ill patients undergoing mechanical ventilation. N Engl J Med 2000, 342:1471-1477.

13. Young D, Harrison DA, Cuthbertson BH, Rowan K, TracMan Collaborators: Effect of early vs late tracheostomy placement on survival in patients receiving mechanical ventilation: the TracMan randomized trial. JAMA 2013, 309:2121-2129.

14. Terragni PP, Antonelli M, Fumagalli R, Faggiano C, Berardino M, Pallavicini FB, Miletto A, Mangione S, Sinardi AU, Pastorelli M, Vivaldi N, Pasetto A Della Rocca G, Urbino R, Filippini C, Pagano E, Evangelista A, Ciccone G, Mascia L, Ranieri VM: Early vs late tracheotomy for prevention of pneumonia in mechanically ventilated dult ICU patients: a randomizedcontrolled trial. JAMA 2010, 303:1483-1489.

15. Esteban A, Alía I, Gordo F, Fernández R, Solsona JF, Vallverdú I, Macías S, Allegue JM, Blanco J, Carriedo D, León M, de la Cal MA, Taboada F, Gonzalez De Velasco J, Palazón E, Carrizosa F, Tomás R, Suarez J, Goldwasser RS: Extubation out come after spontaneous breathing trials with T-tube or pressure support ventilation. Spanish Lung Failure Collaborative Group. Am J Respir Crit Care Med 1997, 156:459-465.

16. Brochard L, Rauss A, Benito S, Conti G, Mancebo J, Rekik N, Gasparetto A Lemaire F: Comparison of three methods of gradual withdrawal from ventilatory support during weaning from mechanical ventilation. Am J Respir Crit Care Med 1994, 150:896-903.

17. Fraser GL, Devlin JW, Worby CP, Alhazzani W, Barr J, Dasta JF, Kress JP, Davidson JE, Spencer FA: Benzodiazepine versus nonbenzodiazepine-based sedation for mechanically ventilated, critically ill adults: a systematic review and meta-analysis of randomized trials. Crit Care Med 2013, 41(9 Suppl 1):S30-S38.

18. Barr J, Fraser GL, Puntillo K, Ely EW, Gélinas C, Dasta JF, Davidson JE, Devlin JW, Kress JP, Joffe AM, Coursin DB, Herr DL, Tung A, Robinson BR, Fontaine DK, Ramsay MA, Riker RR, Sessler CN, Pun B, Skrobik Y, Jaeschke R, American College of Critical Care Medicine: Clinical practice guidelines for the management of pain, agitation, and delirium in adult patients in the intensive care unit. Crit Care Med 2013, 41:263-306.

19. Neto AS, Nassar AP Jr, Cardoso SO, Manetta JA, Pereira VG, Espósito DC, Damasceno MC, Slooter AJ: Delirium screening in critically ill patients: a systematic review and meta-analysis. Crit Care Med 2012, 40:1946-1951.

20. Pandharipande PP, Girard TD, Jackson JC, Morandi A, Thompson JL, Pun BT, Brummel NE, Hughes CG, Vasilevskis EE, Shintani AK, Moons KG, Geevarghese SK, Canonico A, Hopkins RO, Bernard GR, Dittus RS, Ely EW, BRAIN-ICU Study Investigators: Long-term cognitive impairment after critical illness. N Engl J Med 2013, 369:1306-1316.

21. Vaschetto R, Cammarota G, Colombo D, Longhini F, Grossi F, Giovanniello A Della Corte F, Navalesi P: Effects of propofol on patient-ventilator synchrony and interaction during pressure support ventilation and neurally adjusted ventilatory assist. Crit Care Med 2014, 42:74-82.

22. Strøm T, Martinussen T, Toft P: A protocol of no sedation for critically ill patients receiving mechanical ventilation: a randomised trial. Lancet 2010, 375:475-480

23. Cavaliere F, Antonelli M, Arcangeli A, Conti G, Costa R, Pennisi MA, Proietti R: A low-dose remifentanil infusion is well tolerated for sedation in mechanically ventilated, critically-ill patients. Can J Anaesth 2002, 49:1088-1094.
24. Epstein SK: Noninvasive ventilation to shorten the duration of mechanical ventilation. Respir Care 2009, 54:198-208.

25. Burns KE, Adhikari NK, Keenan SP, Meade MO: Noninvasive positive pressure ventilation as a weaning strategy for intubated adults with respiratory failure. Cochrane Database Syst Rev 2010, 8, CD004127.

26. Tamrat R, Huynh-Le MP, Goyal M: Non-pharmacologic interventions to improve the sleep of hospitalized patients: A systematic review. J Gen Intern Med 2014, 29:788-795.

doi:10.1186/2049-6958-9-45

Cite this article as: Conti et al:: Sedation and weaning from mechanical ventilation: time for 'best practice' to catch up with new realities? Multidisciplinary Respiratory Medicine 2014 9:45.

\section{Submit your next manuscript to BioMed Central and take full advantage of:}

- Convenient online submission

- Thorough peer review

- No space constraints or color figure charges

- Immediate publication on acceptance

- Inclusion in PubMed, CAS, Scopus and Google Scholar

- Research which is freely available for redistribution

Submit your manuscript at www.biomedcentral.com/submit
C) Biomed Central 